\title{
Nível de complexidade assistencial dos pacientes em uma unidade de internação
}

\author{
Level of complexity of patients in a hospitalization unit \\ Nivel de complejidad asistencial de los pacientes en una unidad de internación
}

\author{
Ana Paula de Brito', Edinêis de Brito Guirardello" \\ ' Universidade Estadual de Campinas, Faculdade de Ciências Médicas, Curso de Pós-Graduação em Enfermagem. \\ (Mestranda) Campinas-SP, Brasil. \\ "Universidade Estadual de Campinas, Faculdade de Ciências Médicas, Departamento de Enfermagem, Curso de Pós- \\ Graduação em Enfermagem. Campinas-SP, Brasil.
}

\author{
Submissão: 23-12-2010 Aprovação: 20-12-2011
}

\begin{abstract}
RESUMO
O estudo objetivou avaliar o nível de complexidade assistencial dos pacientes em uma unidade de internação de um hospital de ensino no interior paulista. Trata-se de um estudo descritivo, que utilizou para a coleta de dados o instrumento "Sistema de classificação de pacientes: proposta de complementação do instrumento", de Fugulin et al., por trinta dias consecutivos. Foram realizadas 1.080 observações, obtidas do registro de 156 pacientes, verificando-se predomínio de pacientes que requerem alta dependência de cuidado. Constataram-se diferenças dos pacientes entre os postos da unidade no perfil, tipo de tratamento e nível de complexidade de cuidado. Conclui-se que há necessidade de reavaliar o quadro de profissionais, como também readequar as demandas de recursos tecnológicos e materiais.
\end{abstract}

Descritores: Avaliação em enfermagem; Administração de recursos humanos em hospitais; Dimensionamento de pessoal; Cuidados de Enfermagem.

\begin{abstract}
The objective of this descriptive study was to evaluate the level of patient`s care complexity in a hospital unit belonging to a school hospital located in São Paulo, Brazil. Data collection was carried out using the "Patient Classification System: a proposal to complement the instrument", by Fugulin et al., during thirty consecutive days. The use of this instrument resulted in 1.080 observations obtained from 156 patients' records. The majority of the patients required high level of care dependency; there were differences among the patient 's wards regarding gender and age, type of treatment and patients' level of care complexity. We concluded that there was a need to evaluate the health care providers in the institution, as well as to make adjustments regarding demands for technological and materials resources.
\end{abstract}

Key words: Nursing assessment; Personnel administration, hospital; Personnel downsizing; Nursing care.

\section{RESUMEN}

El objetivo de este estudio fue evaluar el nivel de complejidad asistencial de los pacientes en una unidad de internación de un hospital universitario en el interior de São Paulo - Brasil. Este estudio descriptivo utilizó el sistema de clasificación de pacientes: una oferta de la complementación del instrumento de Fugulin et al. por 30 días consecutivos. Fueran obtenidas 1080 observaciones a partir de los registros de 156 pacientes con predominio de los pacientes con alta dependencia de cuidado. Fueran encontradas diferencias en el perfil, tipo de tratamiento y el nivel de complejidad del cuidado entre los puestos de la unidad. Se concluye que existe la necesidad de otras evaluaciones del cuadro de profesionales, así como readecuar las demandas de recursos tecnológicos y de materiales.

Palabras clave: Evaluación en enfermería; Administración de personal en hospitales; Reducción de personal; Atención de enfermería.

Extraído de Trabalho de Iniciação Científica, financiado pela Fundação de Amparo à Pesquisa do Estado de São Paulo - FAPESP (Processo nº 08/58185-8), Campinas-SP, Brasil.

\section{AUTOR CORRESPONDENTE Ana Paula de Brito E-mail: anap@fcm.unicamp.br}




\section{INTRODUÇÃO}

Nas instituições hospitalares, o serviço de Enfermagem é fundamental no processo assistencial, e, por esse motivo, torna-se necessária a avaliação contínua do quantitativo de recursos humanos de enfermagem em relação à carga de trabalho atribuída à unidade hospitalar onde esses recursos estão alocados ${ }^{(1)}$

Atualmente, o enfermeiro dispõe de instrumentos que possibilitam avaliar o nível de complexidade de cuidado ou a carga de trabalho de Enfermagem requerida pela clientela por meio do Sistema de Classificação de Paciente (SCP), essencial para o dimensionamento. O SCP compreende a classificação de pacientes em grupos de cuidados ou categorias e a quantificação dessas categorias como uma medida dos esforços de Enfermagem requeridos ${ }^{(2)}$.

No Brasil, encontram-se disponíveis instrumentos de classificação para avaliar pacientes adultos ${ }^{(3-7)}$ e pediátricos ${ }^{(8)}$. Todos têm como finalidade a classificação dos pacientes segundo a complexidade do cuidado, ainda que utilizem diferentes maneiras para realizar a classificação.

$\mathrm{O}$ instrumento desenvolvido por Perroca ${ }^{(3)}$ tem como finalidade a classificação de pacientes e foi elaborado tendo por base as necessidades individualizadas de cuidado. É composto por 13 indicadores críticos de cuidado e possibilita classificar os pacientes em quatro categorias de cuidado: cuidados mínimos, intermediários, semi-intensivos e intensivos, obtidas pela somatória dos pontos atribuídos a cada indicador crítico.

Martins e Haddad (4) desenvolveram um instrumento que possibilita classificar pacientes em quatro classes distintas de acordo com o grau de dependência de cuidado. Para ser classificado em uma delas, o paciente deve apresentar um mínimo de três características descritas em uma das classes denominadas por I, II, III e IV.

$\mathrm{O}$ instrumento desenvolvido por Fugulin et al. ${ }^{(5)}$ aborda nove áreas de cuidado que são avaliadas em cinco níveis ou categorias de complexidade assistencial: cuidados mínimos, intermediários, de alta dependência, semi-intensivos e cuidados intensivos. Na segunda versão deste instrumento, houve readequação quanto à forma de classificação e layout, em que se atribui uma pontuação para cada uma das nove áreas de cuidado ${ }^{(6)}$.

A última versão deste instrumento é denominada Sistema de Classificação de Pacientes: proposta de complementação do instrumento de Fugulin et al., com o acréscimo de três áreas de cuidado: a) integridade cutâneo-mucosa/ comprometimento tecidual, b) curativo e c) tempo utilizado na realização de curativos. Entretanto, segue os mesmos parâmetros para a classificação dos pacientes. Os autores destacam que o acréscimo dessas áreas de cuidado permite que o instrumento esteja adequado para identificar diferentes clientelas, principalmente em unidades cirúrgicas ${ }^{(7)}$.

$\mathrm{O}$ instrumento de classificação de pacientes pediátricos ${ }^{(8)}$ (ICPP) tem como finalidade classificar pacientes pediátricos em seu nível de complexidade por meio de 11 indicadores de demanda de Enfermagem que recebem pontuação de um a quatro, em ordem crescente de complexidade. A somatória desses pontos permite classificar os pacientes em cinco categorias: cuidados mínimos, intermediários, autocuidado, cuidados semi-intensivos e cuidados intensivos.

Nos últimos anos, houve uma mudança no perfil de complexidade de cuidado dos pacientes na unidade em estudo. Uma das razões foi o aumento da população idosa com problemas crônicos de saúde, como os cardiovasculares. Assim, a classificação de pacientes em nível de complexidade assistencial torna-se fundamental, não somente para reavaliar a demanda de pessoal de Enfermagem para prestação da assistência, como para identificar outros aspectos da demanda de cuidados que variam segundo a sazonalidade e a complexidade de cuidado que esses pacientes requerem. O presente estudo objetiva avaliar o nível de complexidade de cuidado requerido pelos pacientes em uma unidade de internação.

\section{MÉTODO}

Trata-se de um estudo descritivo, realizado em uma unidade de internação de um hospital de ensino do interior do Estado de São Paulo, de atendimento terciário e quaternário. A unidade contém 36 leitos, divididos em dois postos (P1 e P3) e atende pacientes de diferentes especialidades médicas. O posto 1 contém 18 leitos de clínica médica e o posto 3 contém, quatro leitos de clínica médica, nove de cirurgia vascular, quatro de dermatologia e um de endocrinologia.

A população do estudo foi composta por pacientes internados em ambos os postos da unidade, com idade igual ou superior a 18 anos, independente do diagnóstico médico e tipo de tratamento.

Para a coleta de dados, utilizou-se o instrumento Sistema de classificação de pacientes: proposta de complementação do instrumento de Fugulin et al. ${ }^{(7)}$, com o objetivo de avaliar a complexidade assistencial dos pacientes. É composto por 12 áreas de cuidado: a) estado mental, b) sinais vitais, c) oxigenação, d) motilidade, e) deambulação, f) alimentação, g) eliminação, h) cuidados corporais, i) terapêutica, j) integridade cutâneo mucosa / comprometimento tecidual, k) curativo e l) tempo utilizado na realização de curativos.

Cada uma dessas áreas recebe uma pontuação de um a quatro pontos e a somatória desses pontos pode variar de 12 a 48, indicando de forma crescente a complexidade assistencial do paciente, que corresponde a: cuidado mínimo (12 a 17 pontos), cuidado intermediário (18 a 22 pontos); alta dependência (23 a 28 pontos); cuidado semi-intensivo (29-34 pontos) e cuidado intensivo (34 a 48 pontos).

Para a caracterização clínica e demográfica dos pacientes utilizou-se uma planilha elaborada pelas pesquisadoras e na descrição dos diagnósticos médicos, considerou-se o motivo principal da internação, classificados segundo o Código Internacional de Doenças (CID X) ${ }^{(9)}$.

Previamente à coleta de dados, realizou-se um pré-teste com o instrumento para a familiarização com o mesmo. Foram 30 dias consecutivos de coleta, sempre no mesmo período, por meio do exame físico e avaliação dos registros de Enfermagem e consulta aos prontuários para obtenção de dados clínicos e demográficos dos pacientes. Na aplicação do instrumento, a unidade de medida adotada foi os leitos, 
independente dos pacientes ali alocados. Sendo assim, um mesmo leito pode ter sido utilizado por diferentes pacientes ao longo do período de coleta.

O projeto obteve aprovação do Comitê de Ética em Pesquisa (Parecer $n^{\circ}$ 876/2008) com dispensa da assinatura do Termo de Consentimento Livre e Esclarecido (TCLE), pois a avaliação das demandas de cuidado junto ao paciente é considerada uma atividade cotidiana do Enfermeiro e os pacientes não foram submetidos a nenhum outro procedimento em função da aplicação do instrumento.

Os dados foram armazenados em uma planilha eletrônica no programa Microsoft ${ }^{\circledast}$ Excel e analisados com o auxílio do SPSS $^{\circledast} 16.0$ for Windows. Foram feitas tabelas de freqüência das variáveis categóricas e estatísticas descritivas (média, desvio padrão, mediana, mínimo e máximo) das variáveis contínuas.
Para verificar a existência de associação entre os postos com relação às variáveis categóricas, foi utilizado o Teste Qui-quadrado e, quando os valores esperados foram menores que cinco, utilizou-se o Teste Exato de Fisher. O nível de significância adotado foi de 5\%, ou seja, p-valor $\leq 0,05$.

\section{RESULTADOS E DISCUSSÃO}

A amostra foi composta por 156 pacientes, na maioria do sexo masculino $(60,3 \%)$, idade média de 54,9 anos $(\mathrm{min}=18$ e $\max =90)$ e tempo médio de internação de 10,32 dias $(\min =1$ e $\max =39)$. Quanto à procedência, 33,3\% vieram do setor de internação; $32 \%$ da unidade de emergência referenciada; $12,8 \%$ de outras enfermarias; $9 \%$ dos ambulatórios; $6,4 \%$ do centro cirúrgico; $5,1 \%$ da unidade de terapia intensiva e $1,3 \%$ transferidos de outros hospitais (Tabela 1 ).

Tabela 1 - Dados demográficos e clínicos dos pacientes internados na Unidade de Internação. Campinas-SP, 2009.

\begin{tabular}{|c|c|c|c|}
\hline \multirow{2}{*}{ VARIÁVEIS } & POSTO 1 & POSTO 3 & \multirow[t]{2}{*}{ p - valor } \\
\hline & n (\%) & n (\%) & \\
\hline Gênero & & & $0,016^{*}$ \\
\hline Masculino & $29(48,3)$ & $65(67,7)$ & \\
\hline Feminino & $31(51,7)$ & $31(32,3)$ & \\
\hline Idade & & & $0,048^{*}$ \\
\hline$<30$ & $10(16,6)$ & $10(10,4)$ & \\
\hline $31-40$ & $10(16,6)$ & $8(8,3)$ & \\
\hline $41-50$ & $5(8,3)$ & $7(7,2)$ & \\
\hline $51-60$ & $16(26,6)$ & $17(17,7)$ & \\
\hline$>60$ & $19(31,6)$ & $54(56,2)$ & \\
\hline Precedência & & & $<0,001 * *$ \\
\hline Serviço de Internação & $3(5,0)$ & $49(51,0)$ & \\
\hline Unidade Emergência Referenciada & $34(57,0)$ & $16(17,0)$ & \\
\hline Enfermarias & $9(15,0)$ & $11(11,0)$ & \\
\hline Ambulatórios & $11(18,0)$ & $3(3,0)$ & \\
\hline Centro Cirúrgico & 0 & $6(6,0)$ & \\
\hline Unidade de terapia Intensiva & $2(3,0)$ & $10(10,0)$ & \\
\hline Outros hospitais & $1(2,0)$ & $1(1,0)$ & \\
\hline Tratamento & & & $<0,001 *$ \\
\hline Clínico & $57(95,0)$ & $25(26,0)$ & \\
\hline Cirúrgico & $3(5,0)$ & $71(74,0)$ & \\
\hline Tempo de Internação & & & $<0,001 * *$ \\
\hline Médio & 12,5 & 8,14 & \\
\hline Mínimo & 4 & 1 & \\
\hline Máximo & 39 & 25 & \\
\hline Principais Diagnósticos***: & & & $<0,001 * *$ \\
\hline Doenças do Aparelho. Circulatório & $13(21,7)$ & $58(61,7)$ & \\
\hline Doenças do Aparelho. Respiratório & $12(20,0)$ & $4(4,3)$ & \\
\hline Neoplasias & $3(5,0)$ & $8(8,5)$ & \\
\hline Doenças Endócrinas & $6(10,0)$ & $4(4,3)$ & \\
\hline Doenças da Pele e Tecido Subcutâneo & $5(8,3)$ & $4(4,3)$ & \\
\hline
\end{tabular}

Legenda:

* Teste Qui-Quadrado

** Teste Exato de Fisher

*** Classificação segundo o Código Internacional de Doenças (CID X) 
Os diagnósticos médicos mais freqüentes, segundo o CID X foram: doenças do aparelho circulatório $(45,5 \%)$, aparelho respiratório $(10,3 \%)$, neoplasias $(7,1 \%)$, doenças endócrinas, nutricionais e metabólicas $(6,4 \%)$ e doenças da pele e tecido celular subcutâneo $(5,8 \%)$. Do total de pacientes, $52,5 \%$ haviam sido submetidos a tratamentos clínicos e $47,5 \%$ a tratamentos cirúrgicos (Tabela 1).

O local de procedência da maioria dos pacientes do Posto 1 foi a unidade de emergência referenciada (UER), pois este Posto aloca pacientes para investigação diagnóstica e compensação clínica que dão entrada ao hospital pela UER, enquanto o Posto 3 recebe a maioria dos pacientes procedentes do serviço de internação, pois tem seus leitos destinados a especialidades cirúrgicas, com internação eletiva para esses procedimentos.

A caracterização do perfil demográfico indicou um maior número de pacientes internados no Posto 3, que apresenta maior rotatividade, pois os pacientes são submetidos a procedimentos que requerem períodos de internação inferiores a seis dias, nas especialidades médicas de dermatologia, endocrinologia e oftalmologia.

As doenças encontradas com maior freqüência foram as do sistema circulatório, respiratório e neoplasias. Esse perfil de doenças crônico-degenerativo era esperado na Unidade em estudo, pois acomete principalmente idosos que são a maioria dos pacientes ali assistidos ${ }^{(10-11)}$.

Ao avaliar se existiam diferenças quanto ao perfil de pacientes entre os Postos, foi possível constatar que o Posto 3 apresenta um predomínio de pacientes do sexo masculino $(p=0,016)$, com faixa etária superior a 60 anos $(p=0,048)$, submetidos a tratamentos cirúrgicos $(p=0,001)$, com doenças do aparelho circulatório e provenientes do setor de internação $(p=0,001)$.

O predomínio de pacientes do sexo masculino e idosos no Posto 3 está relacionado aos diagnósticos médicos mais freqüentes em cada Posto. No posto 3 estão os pacientes com diagnósticos de doenças do aparelho circulatório que acometem majoritariamente os homens ${ }^{(12)}$ e que são patologias crescentes na população idosa atual ${ }^{(10)}$.

O instrumento foi aplicado durante 30 dias consecutivos em ambos os Postos, totalizando em 1080 observações, obtidas do registro dos 156 pacientes. Destaca-se que o número de observações não corresponde ao número total de pacientes, pois um mesmo paciente pode ter sido classificado mais de uma vez, segundo o tempo de permanência na Unidade (Tabela 2).

Houve um predomínio de pacientes que requeriam cuidados de alta dependência, seguidos de cuidados intermediários e semi-intensivos. Esse perfil de complexidade não era esperado para a Unidade em estudo, que tem estrutura para atender pacientes de cuidados mínimos e intermediários, e não de alta complexidade como o encontrado. Tal panorama revela problemas na alocação dos pacientes na instituição, pois se trata de um hospital de ensino de nível terciário e quaternário, que tem um constante aumento da complexidade dos pacientes atendidos e que não é acompanhado de melhoria da infraestrutura adequada para atendê-los.
Tabela 2 - Descrição dos níveis de complexidade assistencial dos pacientes nos dois postos da Unidade de Internação. Campinas-SP, 2009.

\begin{tabular}{lcccc}
\hline \multirow{2}{*}{$\begin{array}{c}\text { CATEGORIA DE } \\
\text { CUIDADO }\end{array}$} & \multicolumn{4}{c}{ Unidade de Internação } \\
\cline { 2 - 4 } & $\mathrm{N}$ & $\%$ & $\mathrm{~N}$ & $\%$ \\
& & & & \multicolumn{2}{c}{ POSTO 3 } \\
\hline Alta-Dependência & 159 & 29,44 & 159 & 29,44 \\
Intermediário & 142 & 26,30 & 100 & 18,52 \\
Mínimo & 77 & 14,26 & 49 & 9,07 \\
Semi-Intensivo & 59 & 10,93 & 110 & 20,37 \\
Intensivo & 50 & 9,26 & 93 & 17,22 \\
\hline
\end{tabular}

Em um estudo com a aplicação da versão anterior do instrumento, constatou-se que a maior parte dos pacientes encontrava-se no nível de complexidade intermediária ${ }^{(13)} \mathrm{e}$ que, no presente estudo, a maioria foi classificada como cuidado de alta dependência. Em outro estudo com a aplicação desta mesma versão junto à Unidade de Pronto-socorro, a maioria dos pacientes foi classificada em cuidado mínimo (14). Tais diferenças podem ser decorrentes das diferentes versões utilizadas, pois a versão usada no presente estudo contempla três outras áreas de cuidado.

O predomínio de pacientes que requerem cuidados de alta-dependência na Unidade de Internação justifica-se pelo grande número de pacientes idosos internados, que se enquadram nessa categoria de cuidados devido às incapacidades físicas, cognitivas e emocionais o que resulta em grande dependência da equipe de Enfermagem para o atendimento de suas necessidades.

Os níveis de complexidade assistencial predominantes no Posto 1 são cuidados mínimos e intermediários, enquanto no Posto 3 predominam cuidados semi-intensivos e intensivos. O percentual de cuidados de alta dependência é igual para ambos os postos.

O Posto 3 apresenta maior porcentagem de pacientes com complexidade elevada. Uma das justificativas para esse achado é que neste Posto concentram-se os pacientes com problema vascular, em sua maioria, idosos submetidos à amputação de membros ${ }^{(12,15)}$ e que, consequentemente, necessitam de maior tempo de Enfermagem para cuidados na mobilização, realização de curativos, bandagens e alívio da dor.

Observou-se que em quatro leitos da Unidade os pacientes foram classificados em sua maioria, como cuidados semi-intensivos e intensivos. Esses são os leitos que possuem sistema de gerador de energia e que estão localizados próximos ao posto de Enfermagem, sendo, portanto, mais apropriados para alocar pacientes com necessidades de monitoramento, ventilação mecânica, drogas vasoativas, entre outros, o que justifica o nível de complexidade neles encontrado. 
Frente a esse perfil de complexidade de cuidado, emerge a necessidade de reavaliar a estrutura da unidade para atender essa demanda de cuidados, desde os recursos materiais e tecnológicos, como o quantitativo do quadro de profissionais da unidade e sua qualificação profissional, condições indispensáveis para a assistência com segurança desse perfil de pacientes, que necessita de assistência de Enfermagem constante e especializada, bem como para a segurança dos profissionais que realizam o cuidado.

Outra questão fundamental está na alocação desses pacientes dentro da instituição, pois deveriam ser assistidos em unidades de cuidados semi-intensivos ou intensivos, que possuem recursos humanos, materiais e tecnológicos apropriados para esse perfil de pacientes.

\section{CONCLUSÕES}

O presente estudo possibilitou avaliar o grau de complexidade assistencial dos pacientes assistidos na unidade de internação, revelando que há um predomínio daqueles que necessitam de cuidados de alta dependência, seguido dos que requerem cuidados intermediários e semi-intensivos, o que corresponde ao perfil dos internados nessa unidade, que atende predominantemente idosos acometidos por doenças crônico-degenerativas.

Diante do aumento do nível de complexidade de cuidado apresentado pelos pacientes, torna-se necessário reavaliar tanto o quadro de profissionais que prestam assistência na Unidade, como também readequar as demandas de recursos tecnológicos e materiais.

\section{REFERÊNCIAS}

1. Fugulin FMT. Dimensionamento de pessoal de enfermagem: avaliação do quadro de pessoal das unidades de internação de um hospital de ensino. São Paulo. Tese [Doutorado em Ciências da Saúde] - Escola de Enfermagem da Universidade de São Paulo; 2002.

2. Giovannetti P. Understanding patient classification systems. J Nurs Adm 1979;9(2):4-9.

3. Perroca MG, Gaidzinski RR. Sistema de classificação de pacientes: construção e validação de um instrumento. Rev Esc Enferm USP 1998;32(2):153-68.

4. Martins EAP, Haddad MCL. Validação de um instrumento que classifica os pacientes em quatro graus de dependência do cuidado de enfermagem. Rev Latinoam Enferm 2000;8(2):74-82.

5. Fugulin FMT, Silva SHS, Shimizu HE, Campos FPF. Implantação do sistema de classificação de pacientes na unidade de clínica médica do hospital universitário da USP. Rev Med HU-USP 1994; 4(1/2):63-8.

6. Fugulin FMT, Gaidzinski RR, Kurcgant P. Sistema de classificação de pacientes: identificação do perfil assistencial dos pacientes das unidades de internação do HU-USP. Rev Latinoam Enferm 2005;13(1):72-8.

7. Santos F, Rogenski NMB, Baptista CMC, Fugulin FMT. Sistema de classificação de pacientes: proposta de complementação do instrumento de Fugulin et al. Rev Latinoam Enferm 2007; 15(5):980-5.
8. Dini AP. Sistema de classificação de pacientes pediátricos: construção e validação de instrumento. .Campinas-SP. Dissertação [Mestrado em Ciências da Saúde] - Universidade Estadual de Campinas; 2007.

9. Departamento de Informática do SUS [homepage na internet]. Classificação Internacional de Doenças. [acesso em 29 jun 2009]. Disponível em: http://www.datasus.gov. br/cid10/v2008/cid10.htm.

10. Simões CCS. Perfis de Saúde e de Mortalidade no Brasil: uma análise de seus condicionantes em grupos populacionais específicos. Brasília : OPAS; 2002.

11. Veras R. Envelhecimento Populacional Contemporâneo: demandas, desafios e inovações. Rev Saúde Pública 2009;43(3): 548-54.

12. Menezes FH, Luccas GC, Lane JC. Manual de Moléstias Vasculares. 1a ed. Rio de Janeiro: AC Farmacêutica; 2009.

13. Fakih FT, Carmagnani MSI, Cunha ICKO. Dimensionamento de pessoal de enfermagem em um hospital de ensino. Rev Bras Enferm 2006;59(2):183-7

14. Ohara R, Melo MRAC, Laus AM. Caracterização de perfil assistencial dos pacientes adultos de um pronto socorro. Rev Bras Enferm 2010;63(5):749-54.

15. Agne JE, Cassol CM, Bataglion D, Ferreira FV. Identificação das Causas de Amputação de Membros no Hospital Universitário de Santa Maria. Saúde (São Paulo) 2004;30(1-2):84-9. 